\title{
How often do we identify fetal abnormalities during routine third-trimester ultrasound? A systematic review and meta-analysis
}

Lior Drukker ${ }^{1,2}$, Bradburn Elizabeth ${ }^{1}$, Gabriel B. Rodriguez ${ }^{1,3}$, Nia Wyn Roberts ${ }^{4}$, Lawrence Impey $^{2}$, Aris T. Papageorghiou ${ }^{1}$

1. Nuffield Department of Women's \& Reproductive Health, University of Oxford, John Radcliffe Hospital, Oxford, United Kingdom

2. Fetal Medicine Unit, Department of Maternal and Fetal Medicine, Women's Center, John Radcliffe Hospital, Oxford University Hospitals NHS Foundation Trust, Oxford, United Kingdom

3. College of Public Health, Department of Epidemiology and Biostatistics, University of Georgia, Georgia, United States of America

4. Bodleian Health Care Libraries, University of Oxford, Oxford, United Kingdom

Short title: Third-trimester anomalies systematic review

\section{Corresponding author}

Prof. Aris Papageorghiou

This article has been accepted for publication and undergone full peer review but has not been through the copyediting, typesetting, pagination and proofreading process, which may lead to differences between this version and the Version of Record. Please cite this article as doi: 
Nuffield Department of Women's \& Reproductive Health, John Radcliffe Hospital, University of Oxford, Oxford OX3 9DU

E: aris.papageorghiou@wrh.ox.ac.uk

T: $+44(0) 1865221019$ 


\section{ABSTRACT}

Background Routine third-trimester ultrasound is frequently offered to pregnant women to identify fetuses with abnormal growth. Infrequently, a congenital anomaly is incidentally detected.

Objective To establish the prevalence and type of fetal anomalies detected during routine thirdtrimester scans using a systematic review and meta-analysis.

Search strategy Electronic databases (MEDLINE, EMBASE, and the Cochrane library) from inception until August 2019.

Selection criteria Population-based studies (randomized control trials, prospective and retrospective cohorts) reporting abnormalities detected at the routine third-trimester ultrasound performed in unselected populations with prior screening. Case reports, case series, case-control studies and reviews without original data were excluded.

Data collection and analysis Prevalence and type of anomalies detected in the third trimester. We calculated pooled prevalence as the number of anomalies per 1,000 scans with $95 \%$ confidence intervals (95\% CI). Publication bias was assessed.

Main Results The literature search identified 9,594 citations; thirteen studies were eligible representing 141,717 women; 643 were diagnosed with an unexpected abnormality. The pooled prevalence of a new abnormality diagnosed was 3.68 per 1,000 (95\% CI 2.72 - 4.78) women scanned. The largest groups of abnormalities were urogenital (55\%), central nervous system abnormalities (18\%) and cardiac abnormalities (14\%).

Conclusion Combining data from 13 studies and over 140,000 women, we show that during routine third trimester ultrasound, an incidental fetal anomaly will be found in about 1 in 300 scanned women. This information should be taken into account when consenting women for third trimester ultrasound; and when designing and assessing cost of third trimester ultrasound screening programs.

\section{Source of Funding}

ATP and LD are supported by the National Institute for Health Research (NIHR) Oxford Biomedical Research Centre (BRC). The views expressed are those of the author(s) and not necessarily those of the NHS, the NIHR or the Department of Health. The study itself was not funded. 
Keywords: pregnancy care, congenital malformation, fetal anomaly, fetal abnormality, anomaly scan, third-trimester, incidental finding, systematic review

Tweetable abstract: One in 300 women attending a third-trimester scan will have a finding of a fetal abnormality. 


\section{INTRODUCTION}

Routine third-trimester growth scans in at low-risk women are increasingly recommended with the aim of increasing the detection of small for gestational age fetuses, large-for-gestational-age fetuses $^{1-4}$, and breech presentation ${ }^{5,6}$, all conditions associated with a higher risk of adverse perinatal outcome ${ }^{7-11}$. Nevertheless, there is an ongoing debate as to whether routine late pregnancy ultrasound in low-risk populations confers benefits on the mother or the baby ${ }^{12-15}$.

Uncommonly, during a routine third-trimester scan, a previously undiagnosed fetal congenital abnormality is detected. Broadly speaking, this includes two separate groups: first, it includes congenital defects that were present earlier, but not detected despite adherence to first- and second-trimester screening programs; and second, structural abnormalities that develop or manifest only in late pregnancy and could not have reasonably been detected earlier. The abnormalities included in the first group are those not identified earlier due to unfavourable maternal habitus or fetal lie; ones that were overlooked by the screening sonologist; and also conditions that may be more easily visualized in a larger fetus, such as a ventricular septal defect (VSD). The second group includes abnormalities that can only be seen with fetal maturation, such as certain malformations of cortical development, microcephaly or hydrocephaly; gastrointestinal abnormalities relating to intestinal obstruction; urinary tract abnormalities that change over time such as renal pelvis dilatation; and some skeletal dysplasias. ${ }^{16-20}$

There are some important considerations when detecting abnormalities in late pregnancy. These include factors at the level of the individual, such as ensuring appropriate consent prior to growth scans, anxiety provoked given the limited time to carry out full investigations, and referral for appropriate fetal / neonatal care; and implications at the level of the health system, such as the need for efficient late referral pathways; associated costs of growth screening In general, even late diagnosis of an abnormality will allow parents to prepare for birth of a baby with an anomaly, timely in-utero transfer, and guide appropriate follow up of the newborn and infant.

Because of this, estimating the prevalence of finding such anomalies at the time of the routine growth scan is of relevance to patients, practitioners and healthcare providers, as well as researchers planning third trimester studies. Data on detection are not well established and are also dependent on the type of protocol used, for example, whether a third trimester is intended to carry 
out only an assessment of fetal growth / amniotic fluid / presentation ${ }^{21}$ or whether it is intended to also formally reassess fetal anatomy ${ }^{22}$.

The aim of this study was to establish the prevalence and determine the type of malformations (chromosomal, genetic, and structural) that are detected at the time of routine third-trimester ultrasound screening for fetal growth in populations with adequate prior screening.

\section{METHODS}

This is a systematic review and meta-analysis, conducted in accordance with the recommended Preferred Reporting Items for Systematic Reviews and Meta-Analyses (PRISMA) guidelines and the Synthesizing Evidence from Diagnostic Accuracy Tests (SEDATE) guidance ${ }^{23,24}$. Patients were not involved in the development of this study.

\section{Literature search}

The following databases were searched electronically (by the librarian N.W.R) for relevant citations, from January 1980 until 28 August 2019: MEDLINE (OvidSP)[1946-present], EMBASE(OvidSP)[1974-present], Cochrane Database of Systematic Reviews(Cochrane Library, Wiley)[Issue 8 of 12, August 2019], Cochrane Central Register of Controlled Trials(Cochrane Library, Wiley)[Issue 8 of 12, August 2019], Database of Abstracts of Reviews of Effects(Cochrane Library, Wiley)[issue 2 of 4, April 2015] and Science Citation Index \& Conference Proceedings Citation Index(Web of Science Core Collection)[1945-present]. The search strategy consisted of relevant MeSH terms, keywords and word variants for 'malformation' 'third-trimester' and 'ultrasound,' restricted to English (Appendix S1). Reference lists of relevant articles were searched manually to identify papers not found using the electronic searches.

\section{Eligibility criteria}

We included all population-based studies (randomized control trials, prospective cohort, retrospective cohort) that undertook routine third trimester ultrasound. To ensure unbiased results which reflect usual practice, we included those studies that reported on unselected pregnant populations that had previous ultrasound screening, and that reported on all fetal malformations (structural, chromosomal, genetic) identified for the first time at this routine third-trimester ultrasound scan. For randomized controlled trials, we included only those women that were in the study arm which included a third-trimester scan. 
Studies were excluded if they: (1) did not include a third-trimester scan, or where this was not done routinely (the third-trimester scan was recognized as routine only if this was specifically acknowledged); (2) reported malformations detected in selected populations, such as in referred women, or high-risk pregnancies alone (e.g., in women with gestational diabetes); (3) were case reports, case series, case-control studies, and reviews or meta-analyses without original data (4) had a policy of no previous second-trimester anomaly screening; (5) did not report both the type or and number of malformations found at the third-trimester (6) reported only on malformations of a specific system, or having ultrasound scans dedicated to a specific system (such as a study assessing only the fetal urinary system).

\section{Study Selection}

Two researchers (L.D and E.B) independently screened the titles and abstracts of all identified citations and selected potentially eligible studies. We then retrieved and independently assessed these studies for inclusion and data extraction. Discrepancies on assessments of elements or on the inclusion of the papers between reviewers were resolved by discussion with A.T.P.

\section{Data Extraction}

Data were extracted from each article using a specially designed data-extraction spreadsheet. The following data were captured: study characteristics (authors, year of publication, study design, healthcare settings, study period), population characteristics (inclusion and exclusion criteria), ultrasound scan schedule (first, second, and third-trimester gestational ages), ultrasound scan guidelines (scan protocol and checklist of each scan), number of women attending the thirdtrimester scan (sample size), malformations detected before the third trimester, at the third trimester and after birth (type of malformations included, time of detection, proportion detected), as well as detailed information on malformations detected at the third-trimester scan (number identified, affected systems, and type of malformations). With respect to the third-trimester scan protocol, studies were classified as performing a growth scan (three standard biometric planes), following a prespecified anatomy checklist of at least one organ, or performing a repeat detailed assessment of the fetal anatomy in a systematic way.

All types of congenital malformations, including structural, genetic, and chromosomal, either minor or major, reported in each individual cohort were included in the current analysis. Isolated abnormalities of fetal growth, umbilical cord, amniotic fluid, membranes, or placenta without 
evidence of a congenital fetal malformation, were beyond the scope of the current analysis. In order to reduce the risk of error, all data were collected and extracted from tables independently by two authors (L.D and E.B).

Data regarding the number of false-positive diagnoses made in the third-trimester were not extracted since for many anomalies it is not possible to distinguish false-positive diagnoses from changes due to natural history.

\section{Quality assessment}

Assessment of the quality of the studies included in this review was performed using the Quality Assessment of Diagnostic Accuracy Studies (QUADAS-2) ${ }^{25}$, a tool designed to evaluate the risk of bias within each study and assess its applicability to the systematic review. Studies were assessed within the four key domains of patient selection, index test, reference standard and flow of patients through the study, along with the timing of the index test. A judgment of low, high or unclear risk of bias and lack of applicability was made for each study by assessing each domain with respect to bias, and the first three domains with respect to applicability, based on a series of signaling questions developed specifically to our review (Appendix S2).

\section{Data Analysis}

Descriptive tables were produced for study characteristics, ultrasound screening strategy, and malformations detected in the third-trimester for the included studies. For each study we calculated the prevalence of malformations identified, and the proportion of women attending the third-trimester scan where a malformation is detected (and the $95 \%$ confidence intervals $(95 \%$ CI)).

The main outcomes were the prevalence and type of anomalies detected at the third trimester ultrasound scan. Subgroup analysis by scan period (by decade) was carried out to calculate the pooled prevalence in each decade group. Pooled prevalence is expressed as the number of anomalies per 1,000 scans with 95\% CI. Publication bias was assessed with a funnel plot, and we tested for asymmetry in the funnel plot using an unweighted Egger's test. Statistical analyses were carried out in version 3.6.2 of R using the "Meta" and "Metafor" packages to create the analysis and plots used in this paper.

\section{RESULTS}




\section{Literature identification}

The initial search identified 9,594 citations. The full text of 310 manuscripts was reviewed for eligibility and 297 were subsequently excluded as they did not meet the selection criteria. Overall, 13 studies were selected for inclusion in the systematic review ${ }^{21,22,26-36}$ (Figure 1).

\section{Study Characteristics}

The studies evaluated were published across four decades, and conducted in China, Europe, India, and the USA. Nine out of thirteen studies were single-centre studies, three were two-centre studies $^{21}$,22, 30 and one was multi-centre ${ }^{27}$. Of the included studies, there were three randomized controlled trials, for which only the screening/third-trimester scan arm was included in the review, and there were six prospective studies. Five studies were conducted before 2000. Five studies did not offer a first-trimester scan as part of routine care (unclear in one study). A routine secondtrimester scan was an essential part of our inclusion criteria and was therefore carried out for all women included in the selected studies. These and other study characteristics are detailed in Table 1. There were differences in the gestational age of the third-trimester scan, ranging from as early as 28 weeks or as late as 40 weeks. In the majority of studies, the third-trimester scan was a repeat anomaly scan (7/13 studies). Additionally, there were two studies that specified that they offered a growth scan in the third-trimester, and four studies that did not specify the protocol of the thirdtrimester scan, i.e., it was not clear whether scans were undertaken only for growth, or also for anomaly detection.

\section{Prevalence and type of malformations detected in the third-trimester}

In the 13 included studies, a total of 141,717 women underwent a routine third-trimester scan (Table 2). The median number of women included per study was 7575 (range $808-52,713$ ). In these women, a total of 643 fetuses with congenital malformation/s were identified for the first time during the third-trimester scan. In the 13 studies, the number of fetal abnormalities detected at the third-trimester scan (as a proportion of all fetal abnormalities detected prenatally) varied widely, from $0 \%$ to $24.8 \%$.

The pooled prevalence of a newly diagnosed malformation at a third-trimester ultrasound was 3.68 (95\% CI 2.72 - 4.78) in 1,000 scans (Figure 2). Sub analysis of the studies by the scan protocol revealed that the combined prevalence was 4.20 (95\% CI $3.81-4.61)$ per 1,000 scans for the seven studies whose protocol was repeat anomaly scan and 2.76 (95\% CI $1.91-3.75)$ per 1,000 
scans for the two studies that specifically mention only conducting a growth scan. In four studies, the third-trimester scan protocol was unspecified. Subgroup analysis by scan period (decade) showed no significant difference in the prevalence of anomalies (Figure S1).

The overall observed heterogeneity of the included studies in our analysis was high $\left(\mathrm{I}^{2}=88 \%\right)$. Sensitivity analyses found that the estimated prevalence is not different at a statistically significant level (Table S1).

We found that the funnel plot (Figure S2) was statistically asymmetrical at a significant level $(\mathrm{p}=0.0221)$. This asymmetry does not confirm publication bias, as the distribution on the funnel plot is not skewed to the left or the right. Rather, this asymmetry is more likely due to the high degree of heterogeneity present in the studies.

Anomalies detected at the routine third-trimester scan are listed in Table S2. Genitourinary anomalies were the most common anomaly $(54.6 \%$, 351/643). Within urogenital anomalies, renal pelvic dilatation was the most common anomaly $(57.8 \%, 203 / 351)$, and this condition represented roughly 1 in 3 of all abnormalities detected in the third trimester. Central nervous system anomalies represented the second most common group of abnormalities (17.6\%, 113/643), of which the majority were mild/moderate ventriculomegaly $(49.6 \%, 56 / 113)$. The third most common group was cardiac defects $(14 \%, 90 / 643)$.

\section{Methodological quality assessment of included studies}

Results of the QUADAS-2 assessment are displayed in Figure S3. With respect to bias in patient selection, 9 of the 13 included studies were scored as having a low risk of bias. Four studies were deemed to be at high risk of bias owing to failure to provide adequate information regarding methods used to enroll patients. With respect to the index test, all studies reported the gestational age of the third-trimester scan. Four studies did not specify the third-trimester scanning protocol (i.e., growth, repeat anomaly scan, or other) and were therefore considered at high risk of bias. All studies were found to be at low risk of bias relating to the reference standard, but it should be noted that the risk of bias relating to the reference standard was determined to be low due to our exclusion criteria.

\section{DISCUSSION}

\section{Main findings}


In this study, we have shown that in 3.68 (95\% CI $2.72-4.78)$ out of 1,000 women with prior screening, a previously unrecognized congenital abnormality was diagnosed at a routine thirdtrimester scan. Studies that are explicitly designed to address the detection of unexpected fetal malformations in the third-trimester are rare. Nevertheless, we were able to identify 13 eligible studies reporting previously undetected fetal malformations identified for the first time in the third trimester. Of the included studies, seven carried out a repeat anomaly scan in the third-trimester, two offered a growth scan only, and the third-trimester scan protocol was not clearly described in the remaining four studies. The prevalence of abnormalities detected in those that undertake a growth scan only was 1.5 per 1000 while with repeat anomaly scanning it was 3.8 per 1000 . It should be noted that these may be lower than or higher when taking into account the confidence intervals. In addition, the confidence intervals overlap between these two approaches; nevertheless, it is logical that an approach that purposely looks for anomalies will detect more than a method where they are truly incidental.

About half of the identified malformations in late gestation were of the genitourinary system, with the majority being renal pelvis dilatation, followed by ovarian cysts, duplex kidney/s, unilateral renal agenesis, and polycystic/multicystic kidneys. The second most common system involving malformations detected in the third trimester was the central nervous system, in which mainly ventriculomegaly/hydrocephalus was the most common. The third most common system involving abnormalities was cardiac, in which ventricular septal defect was the most prevalent.

\section{Strengths and limitations}

The present meta-analysis offers an up-to-date, international perspective on newly identified anomalies in the third trimester. We ensure that we included different study designs including randomized control trials, prospective cohort, retrospective cohort that undertook routine third trimester ultrasound from different countries, varying gestational ages, and offering different third-trimester scan protocols were analysed. To ensure that we include all relevant studies in the current analysis, the search methodology employed was one that yielded a large number of titles. During the title read, we encountered many studies designed to assess or report fetal growth in the third trimester, however, most often, anomalies were excluded from these studies. In addition, relatively few studies were population-based, reporting on the number and type of congenital malformations detected; this is an important inclusion criterion introduced to avoid referral bias. 
Limitations of this review include the finding of wide heterogeneity, most likely due to the studies being carried out across four decades, from 1979 to 2019, representing different eras in pregnancy care. Studies also differed in the third-trimester anatomy checklist, gestational age, and definitions (such as the definition of renal pelvis dilatation); it is known that the use and extent of scanning protocols may influence detection rates at other gestations ${ }^{37}$. This needs to be taken into account when extending these results to specific populations. Nevertheless, the prevalence of newly identified malformations was comparable in most studies. The types of malformations detected did differ between studies, with older studies detecting abnormalities that are today commonly detected at the second- and even first-trimester scan. It should be noted that in some of the included studies ultrasound scans were performed by highly trained individuals using top-quality equipment at tertiary centres, and it is likely that less-experienced ultrasound practitioners may not be able to replicate these results. In addition, the included studies were in populations where thirdtrimester scans are undertaken routinely; in settings where scans are undertaken for clinical indications only, many of the abnormalities identified in the included studies would not be detected if the fetus is clinically normally grown and a repeat scan is not carried out.

Some studies included were not designed to report third-trimester malformations but included these as a part of their results ${ }^{33}$. This made detection of appropriate studies to be included challenging, and there is a possibility that, despite meticulous work carried out by the authors, some papers may have been left out. However, the inability to identify all relevant papers is a common limitation of meta-analyses, also known as the "drawer effect" 38 . In addition, English language restriction was imposed. Nevertheless, unlike systematic reviews of treatment effect, where it is imperative that all evidence is found, our aim was to assess prevalence. Therefore, despite these limitations, a large number of pregnancies were included in the analysis, meaning confidence intervals around the prevalence estimate are narrow. In addition, prevalence figures were comparable between studies, and this further increases the confidence that the results are externally valid.

The review does not include analysis of screening characteristics. While this may be seen as a limitation, it was an a priori decision. The reason we decided not to report false-positive rates (which previous studies have already suggested to be low ${ }^{30,39}$ ) was because our aims were not to assess third trimester as a screening test for anomalies, rather to establish how many anomalies may be found during a growth screening program, which is the primary purpose of third trimester 
ultrasound. Many of the included studies do not report their false-positive and false-negative rates; this is partly because "false positives" are not easily defined: for example, hydronephrosis that is present on imaging but that resolves cannot be considered as a "false positive"; rather it is a "true positive" with a natural history that includes a high chance of spontaneous resolution. Falsenegative figures are also difficult to calculate, because detailed post-natal ascertainment of abnormalities is usually carried out only if symptoms are apparent. For example, a small ventricular septal defect undiagnosed in the third trimester (false negative) may well remain undetected after birth in asymptomatic babies, meaning the false negative has become a true negative by virtue of not having a reference test. It is for similar reasons that we cannot provide a sub-analysis by the severity of anomalies.

\section{Interpretation}

The type and prevalence of abnormalities detected in the third-trimester depend on the scan protocol. Currently, there is no internationally agreed policy on whether a third-trimester should be routinely offered, nor on what should be included. In England, third-trimester scans are offered only to at-risk women, and include serial ultrasound measurement of fetal size and assessment of wellbeing with umbilical artery Doppler ${ }^{40}$. The Australasian Society for Ultrasound in Medicine has recently published a third-trimester fetal growth scans reporting template which suggests the data-set to be reported; this includes amniotic fluid assessment, basic biometry, umbilical artery PI and a "limited anatomy survey within the limits of late gestation, fetal lie, and maternal

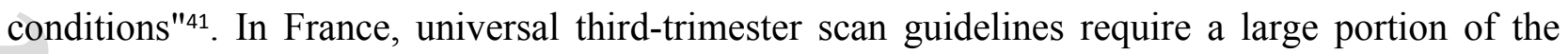
anomaly scan to be repeated ${ }^{42}$. The Società Italiana di Ecografia Ostetrica e Ginecologica e Metodologie Biofisiche questions the utility of routine third-trimester scanning. However, if a third-trimester scan is performed, the following should be evaluated: four-chamber view, stomach, kidneys, and bladder ${ }^{43}$. In Spain, a third-trimester scan is routinely offered to all pregnant women and is limited to the purpose of assessing the amniotic fluid level, fetal presentation, and biometric measurements ${ }^{44}$. In the USA, "Practice Parameter for the Performance of Limited Obstetric Ultrasound Examinations by Advanced Clinical Providers" discussing a third-trimester examination states that "A limited obstetric ultrasound examination does not include an evaluation of fetal anatomy, and in almost all cases, a standard diagnostic or detailed anatomic evaluation of the fetus has been or will be performed during the index pregnancy." 45 
We have shown that half of the abnormalities detected at third-trimester ultrasound are of the urogenital system. The majority of these are renal pelvis dilatation which overall represents 1 in 3 anomalies identified in the third trimester. Previous studies have shown that renal pelvic dilatation is a common third-trimester finding with spontaneous postnatal resolution in a large proportion of the cases $^{46,47}$. The growing kidneys increase in function, produce more urine, and hence pathologies not evident previously can be unmasked. This is an example by which a proportion of the abnormalities identified in the third trimester may result in maternal anxiety and consumption of resources despite being of limited clinical importance. Nevertheless, the diagnosis of abnormalities affecting the renal tract is important as it allows postnatal follow up of babies at risk of chronic and progressive renal disease before a patient presents with symptoms or deteriorating renal function ${ }^{48}$. Detection of other abnormalities, in particular those affecting the heart, may fundamentally alter the care pathewy for the mother and newborn.

The chance of an incidental finding during a routine third-trimester scan is important for women who attend a growth scan; for practitioners; and for policymakers planning resource allocation in the context of implementing a protocol of routine growth scanning. The detection of a severe structural malformation at any gestational age provokes emotional, moral, ethical and legal concerns ${ }^{49}, 50$. Prenatal counseling should provide information to prospective parents on possible interventions, appropriate setting, time and route of delivery as well as the expected postnatal outcomes, immediate and long term ${ }^{51}$. In rare cases, a severe condition that leads to severe incurable physical or cognitive disability is diagnosed as late as the third trimester. Here management will depend not only on parental personal and religious beliefs but also on the legal framework surrounding termination of pregnancy. In some European countries including England ${ }^{52}$, this may be legal at any stage of pregnancy for severe abnormalities ${ }^{53}$. In many other countries, termination of pregnancy is not possible at advanced gestational ages ${ }^{54}$.

Many studies refer to the "third-trimester" as a uniform term, but it is not a uniform period. Scans in the early third-trimester may offer better detection of malformations because the fetal size and bone ossification / shadowing do not pose a difficulty, while late third-trimester scans offer a better timing for detection of late growth aberrations and malpresentation. Although anomalies that occur in late gestation may be more likely to be present, there may also be poorer visualization of the brain, heart, and limbs. In current practice, most third-trimester scans are 
scheduled toward the end of the trimester with the aim of detecting growth aberrations, and therefore, it is likely that a different subset of anomalies will be identified.

Our findings are also important when designing systems for artificial intelligence that assess thirdtrimester biometry. The very low incidence of identifiable abnormalities on the three basic biometric planes means that being unable to detect a standard plane is most likely due to an acquisition difficulty, rather than a fetal abnormality.

\section{Conclusion}

By undertaking an exhaustive review of all relevant studies, we have been able to combine data from 13 studies and over 140,000 women. This has demonstrated that during a routine third trimester ultrasound scan, an incidental fetal anomaly will be found in about 1 in 300 women, despite previous ultrasound screening. The most common anomalies were renal pelvis dilatation in a third of cases and other urological anomalies (half of all cases overall); abnormalities of the CNS, most often ventriculomegaly; and cardiac defects. This information should be taken into account taken by caregivers performing such ultrasound, and women attending such scans should be informed of the small possibility of finding an anomaly. The data should also be available to organizations and centres planning to introduce a routine third-trimester scan. 


\section{Acknowledgments}

None

\section{Disclosure of interests}

ATP is a Senior Scientific Advisor of Intelligent Ultrasound. All other authors declare no competing interests. Completed disclosure of interest forms are available to view online as supporting information.

\section{Contribution to Authorship}

LD: planning, carrying out, analysing and writing

EB: carrying out, analysing and writing

GBR: analysing and writing

NWR: planning, carrying out, and writing

LI: planning, analysing, and writing

ATP: planning, carrying out, analysing and writing

\section{Source of Funding}

ATP and LD are supported by the National Institute for Health Research (NIHR) Oxford Biomedical Research Centre (BRC). The views expressed are those of the author(s) and not necessarily those of the NHS, the NIHR or the Department of Health. The study itself was not funded. 


\section{References}

1. Sovio U, White IR, Dacey A, Pasupathy D, Smith GCS. Screening for fetal growth restriction with universal third trimester ultrasonography in nulliparous women in the Pregnancy Outcome Prediction (POP) study: a prospective cohort study. Lancet. 2015 Nov 21;386(10008):2089-97.

2. Khan N, Ciobanu A, Karampitsakos T, Akolekar R, Nicolaides KH. Prediction of largefor-gestational-age neonate by routine third-trimester ultrasound. Ultrasound Obstet Gynecol. 2019 Jun 25.

3. Sovio U, Moraitis AA, Wong HS, Smith GCS. Universal vs selective ultrasonography to screen for large-for-gestational-age infants and associated morbidity. Ultrasound Obstet Gynecol. 2018 Jun;51(6):783-91.

4. Tarca AL, Hernandez-Andrade E, Ahn H, Garcia M, Xu Z, Korzeniewski SJ, et al. Single and Serial Fetal Biometry to Detect Preterm and Term Small- and Large-for-Gestational-Age Neonates: A Longitudinal Cohort Study. PLoS One. 2016;11(11):e0164161.

5. Wastlund D, Moraitis AA, Dacey A, Sovio U, Wilson ECF, Smith GCS. Screening for breech presentation using universal late-pregnancy ultrasonography: A prospective cohort study and cost effectiveness analysis. PLoS Med. 2019 Apr;16(4):e1002778.

6. De Castro H, Ciobanu A, Formuso C, Akolekar R, Nicolaides KH. Value of routine ultrasound examination at 35-37 weeks' gestation in diagnosis of non-cephalic presentation. Ultrasound Obstet Gynecol. 2019 Oct 31.

7. Ashimi Balogun O, Sibai BM, Pedroza C, Blackwell SC, Barrett TL, Chauhan SP. Serial Third-Trimester Ultrasonography Compared With Routine Care in Uncomplicated Pregnancies: A Randomized Controlled Trial. Obstet Gynecol. 2018 Dec;132(6):1358-67.

8. Beta J, Khan N, Khalil A, Fiolna M, Ramadan G, Akolekar R. Maternal and neonatal complications of fetal macrosomia: systematic review and meta-analysis. Ultrasound Obstet Gynecol. 2019 Apr 2. 
9. Impey L, Murphy D, Griffiths M, Penna L, on behalf of the Royal College of Obstetricians and Gynaecologists. External Cephalic Version and Reducing the Incidence of Term Breech Presentation: Green-top Guideline No. 20a. BJOG. 2017 Jun;124(7):e178-e92.

10. Ego A, Monier I, Skaare K, Zeitlin J. Antenatal detection of fetal growth restriction and stillbirth risk: a population-based case-control study. Ultrasound Obstet Gynecol. 2019 Jul 31.

11. Akolekar R, Panaitescu AM, Ciobanu A, Syngelaki A, Nicolaides KH. Two-stage approach for prediction of small for gestational age neonates and adverse perinatal outcome by routine ultrasound examination at 35-37 weeks' gestation. Ultrasound Obstet Gynecol. 2019 Jul 4.

12. Whitworth M, Bricker L, Mullan C. Ultrasound for fetal assessment in early pregnancy. Cochrane Database Syst Rev. 2015 Jul 14(7):CD007058.

13. Henrichs J, Verfaille V, Jellema P, Viester L, Pajkrt E, Wilschut J, et al. Effectiveness of routine third trimester ultrasonography to reduce adverse perinatal outcomes in low risk pregnancy (the IRIS study): nationwide, pragmatic, multicentre, stepped wedge cluster randomised trial. BMJ. 2019 Oct 15;367:15517.

14. Thornton J. Routine third trimester ultrasound in low risk pregnancy confers no benefit!: FOR: The benefits of routine third-trimester scanning are less clear cut. BJOG. 2016 Jun;123(7):1121.

15. Ray CL, Grange G. Routine third trimester ultrasound in low risk pregnancy confers no benefit!: AGAINST: Arguments for a routine third trimester ultrasound: what the meta-analysis does not show! BJOG. 2016 Jun;123(7):1122.

16. Malinger G, Lerman-Sagie T, Watemberg N, Rotmensch S, Lev D, Glezerman M. A normal second-trimester ultrasound does not exclude intracranial structural pathology. Ultrasound Obstet Gynecol. 2002 Jul;20(1):51-6.

17. John R, D'Antonio F, Khalil A, Bradley S, Giuliani S. Diagnostic Accuracy of Prenatal Ultrasound in Identifying Jejunal and Ileal Atresia. Fetal Diagn Ther. 2015;38(2):142-6. 
18. Schramm T, Gloning KP, Minderer S, Daumer-Haas C, Hortnagel K, Nerlich A, et al. Prenatal sonographic diagnosis of skeletal dysplasias. Ultrasound Obstet Gynecol. 2009 Aug;34(2):160-70.

19. Ramachandrappa S, Kulkarni A, Gandhi H, Ellis C, Hutt R, Roberts L, et al. SHOX haploinsufficiency presenting with isolated short long bones in the second and third trimester. Eur J Hum Genet. 2018 Mar;26(3):350-8.

20. Ismaili K, Hall M, Donner C, Thomas D, Vermeylen D, Avni FE, et al. Results of systematic screening for minor degrees of fetal renal pelvis dilatation in an unselected population. Am J Obstet Gynecol. 2003 Jan;188(1):242-6.

21. Drukker L, Cavallaro A, Salim I, Ioannou C, Impey L, Papageorghiou AT. How often do we incidentally find a fetal abnormality at the routine third-trimester growth scan? A populationbased study. Am J Obstet Gynecol. 2020 Jun 3.

22. Ficara A, Syngelaki A, Hammami A, Akolekar R, Nicolaides KH. Value of routine ultrasound examination at 35-37 weeks' gestation in diagnosis of fetal abnormalities. Ultrasound Obstet Gynecol. 2020 Jan;55(1):75-80.

23. Moher D, Liberati A, Tetzlaff J, Altman DG, Group P. Preferred reporting items for systematic reviews and meta-analyses: the PRISMA statement. BMJ. 2009 Jul 21;339:b2535.

24. Sotiriadis A, Papatheodorou SI, Martins WP. Synthesizing Evidence from Diagnostic Accuracy TEsts: the SEDATE guideline. Ultrasound Obstet Gynecol. 2016 Mar;47(3):386-95.

25. Whiting PF, Rutjes AW, Westwood ME, Mallett S, Deeks JJ, Reitsma JB, et al. QUADAS-2: a revised tool for the quality assessment of diagnostic accuracy studies. Ann Intern Med. 2011 Oct 18;155(8):529-36.

26. Brocks V, Bang J. Routine examination by ultrasound for the detection of fetal malformations in a low risk population. Fetal Diagn Ther. 1991;6(1-2):37-45.

27. Crane JP, LeFevre ML, Winborn RC, Evans JK, Ewigman BG, Bain RP, et al. A randomized trial of prenatal ultrasonographic screening: impact on the detection, management, 
and outcome of anomalous fetuses. The RADIUS Study Group. Am J Obstet Gynecol. 1994 Aug;171(2):392-9.

28. Hernadi L, Torocsik M. Screening for fetal anomalies in the 12th week of pregnancy by transvaginal sonography in an unselected population. Prenat Diagn. 1997 Aug;17(8):753-9.

29. Eik-Nes SH, Salvesen KA, Okland O, Vatten LJ. Routine ultrasound fetal examination in pregnancy: the 'Alesund' randomized controlled trial. Ultrasound Obstet Gynecol. 2000 Jun;15(6):473-8.

30. Romosan G, Henriksson E, Rylander A, Valentin L. Diagnostic performance of routine ultrasound screening for fetal abnormalities in an unselected Swedish population in 2000-2005. Ultrasound Obstet Gynecol. 2009 Nov;34(5):526-33.

31. Abu-Rustum RS, Daou L, Abu-Rustum SE. Role of first-trimester sonography in the diagnosis of aneuploidy and structural fetal anomalies. J Ultrasound Med. 2010 Oct;29(10):144552 .

32. Manegold G, Tercanli S, Struben H, Huang D, Kang A. Is a routine ultrasound in the third trimester justified? Additional fetal anomalies diagnosed after two previous unremarkable ultrasound examinations. Ultraschall Med. 2011 Aug;32(4):381-6.

33. Grande M, Arigita M, Borobio V, Jimenez JM, Fernandez S, Borrell A. First-trimester detection of structural abnormalities and the role of aneuploidy markers. Ultrasound Obstet Gynecol. 2012 Feb;39(2):157-63.

34. Wang L, Wu QQ, Chen Y, Ma YQ, Yao L, Li M. Ultrasound screening of fetal structural abnormalities by standard ultrasound views during the first trimester. Chin Med J (Engl). 2013 Mar;126(5):986-7.

35. Skrastad RB, Eik-Nes SH, Sviggum O, Johansen OJ, Salvesen KA, Romundstad PR, et al. A randomized controlled trial of third-trimester routine ultrasound in a non-selected population. Acta Obstet Gynecol Scand. 2013 Dec;92(12):1353-60.

36. Vijaykumar M, Shailaja M, Nilofar M, Kulkarni N. Detection of structural fetal anomalies in third trimester which usually remains undetected in second trimester. 2017;3:158-62. 
37. Karim JN, Roberts NW, Salomon LJ, Papageorghiou AT. Systematic review of firsttrimester ultrasound screening for detection of fetal structural anomalies and factors that affect screening performance. Ultrasound Obstet Gynecol. 2017 Oct;50(4):429-41.

38. Belfort MA, Clark SL, Saade GR, Kleja K, Dildy GA, Van Veen TR, et al. Hospital readmission after delivery: evidence for an increased incidence of nonurogenital infection in the immediate postpartum period. American Journal of Obstetrics and Gynecology. 2010 2010/01;202(1):35.e1-.e7.

39. Saltvedt S, Almstrom H, Kublickas M, Valentin L, Grunewald C. Detection of malformations in chromosomally normal fetuses by routine ultrasound at 12 or 18 weeks of gestation-a randomised controlled trial in 39,572 pregnancies. BJOG. 2006 Jun;113(6):664-74.

40. Royal College of Obstetricians and Gynaecologists. Small-for-Gestational-Age Fetus, Investigation and Management (Green-top Guideline No. 31) 2013 https://www.rcog.org.uk/globalassets/documents/guidelines/gtg_31.pdf [Accessed 11 March 2020].

41. Australasian Society for Ultrasound in Medicine. Third trimester fetal growth scan reporting template. http://www.asum.com.au/files/public/SoP/Current/Obstetrics_and_Gynaecology/Third-TrimesterFetal-Growth-Scans-Reporting-Template-2019.pdf [Accessed 11 March 2020].

42. Conférence Nationale d'Echographie Obstétricale et Foetale. L'échographie de dépistage prénatal. http://www.cfef.org/archives/bricabrac/cneof/rapportcneof2016.pdf [Accessed 11 March 2020].

43. Società Italiana di Ecografia Ostetrica e Ginecologica e Metodologie Biofisiche. Linee Guida. 2015 [cited; Available from: https://www.sieog.it/category/linee-guida/ [Accessed 11 March 2020]

44. Spanish Society of Gynecology and Obstetrics (SEGO). Control del embarazo normal 2017.https://sego.es/Guias_de_Asistencia_Practica\#perinatal [Accessed 11 March 2020]. 
45. American Institute of Ultrasound in Medicine (AIUM). Practice Parameter for the Performance of Limited Obstetric Ultrasound Examinations by Advanced Clinical Providers. J Ultrasound Med. 2018 Jul;37(7):1587-96.

46. Liu DB, Armstrong WR, 3rd, Maizels M. Hydronephrosis: prenatal and postnatal evaluation and management. Clin Perinatol. 2014 Sep;41(3):661-78.

47. Nef S, Neuhaus TJ, Sparta G, Weitz M, Buder K, Wisser J, et al. Outcome after prenatal diagnosis of congenital anomalies of the kidney and urinary tract. Eur J Pediatr. 2016 May;175(5):667-76.

48. Nguyen HT, Herndon CD, Cooper C, Gatti J, Kirsch A, Kokorowski P, et al. The Society for Fetal Urology consensus statement on the evaluation and management of antenatal hydronephrosis. J Pediatr Urol. 2010 Jun;6(3):212-31.

49. Chervenak FA, McCullough LB, Campbell S. Is third trimester abortion justified? Br J Obstet Gynaecol. 1995 Jun;102(6):434-5.

50. Chervenak FA, McCullough LB. Is third-trimester termination justified? Ultrasound Obstet Gynecol. 2003 Jan;21(1):97-8.

51. Lakhoo K. Fetal counselling for surgical conditions. Early Hum Dev. 2012 Jan;88(1):9-13.

52. Boyd PA, Armstrong B, Dolk H, Botting B, Pattenden S, Abramsky L, et al. Congenital anomaly surveillance in England--ascertainment deficiencies in the national system. BMJ. 2005 Jan 1;330(7481):27.

53. Garne E, Khoshnood B, Loane M, Boyd P, Dolk H, Group EW. Termination of pregnancy for fetal anomaly after 23 weeks of gestation: a European register-based study. BJOG. 2010 May;117(6):660-6.

54. Graham RH, Robson SC, Rankin JM. Understanding feticide: an analytic review. Soc Sci Med. 2008 Jan;66(2):289-300.

55. Drukker L, Noble JA, Papageorghiou AT. Introduction to artificial intelligence in ultrasound imaging in obstetrics and gynecology. Ultrasound Obstet Gynecol. 2020 Jun 12. 
Figure 1 Flowchart of search strategy and selection of studies for inclusion in the systematic review

Figure 2 Forest plot of prevalence of a newly diagnosed fetal abnormality at routine third-trimester scans 
Table 1 Characteristics of studies reporting on detection of fetal malformations detected at routine population-based third-trimester scans

\begin{tabular}{|c|c|c|c|c|c|c|c|}
\hline $\begin{array}{l}\text { Authors } \\
\text { (Publication year) }\end{array}$ & Study title & Study design & Population & Number of centers & Study period & Third-trimester scan protocol & $\begin{array}{l}\text { Third-trimester scan } \\
\text { gestational age (weeks) }\end{array}$ \\
\hline $\begin{array}{l}\text { Brocks et al. } \\
(1991)^{26}\end{array}$ & $\begin{array}{l}\text { Routine examination by ultrasound for the detection of fetal } \\
\text { malformations in a low risk population }\end{array}$ & Prospective & Low risk & One & 1984-1989 & Repeat anomaly & 33 \\
\hline $\begin{array}{l}\text { Crane et al. (1994) } \\
27\end{array}$ & $\begin{array}{l}\text { A randomized trial of prenatal ultrasonographic screening: } \\
\text { Impact on the detection, management, and outcome of } \\
\text { anomalous fetuses }\end{array}$ & Randomized trial & Low risk & $\begin{array}{l}109 \text { practices in } 28 \\
\text { laboratories }\end{array}$ & $1987-1991$ & $\begin{array}{l}\text { Repeat anomaly } \\
\text { (screening group only) }\end{array}$ & $31-35$ \\
\hline $\begin{array}{l}\text { Hernádi et al. } \\
(1997)^{28}\end{array}$ & $\begin{array}{l}\text { Screening for fetal anomalies in the } 12 \text { th week of pregnancy } \\
\text { by transvaginal sonography in an unselected population }\end{array}$ & Prospective & Unselected & One & $1992-1995$ & Not stated & 30 \\
\hline $\begin{array}{l}\text { Eik-Nes et al. } \\
(2000)^{29}\end{array}$ & $\begin{array}{l}\text { Routine ultrasound fetal examination in pregnancy: the } \\
\text { 'Alesund' randomized controlled trial }\end{array}$ & Randomized trial & Unselected & One & $1979-1981$ & $\begin{array}{l}\text { Growth } \\
\text { (screening group only) }\end{array}$ & 32 \\
\hline $\begin{array}{l}\text { Romosan et al. } \\
(2009)^{30}\end{array}$ & $\begin{array}{l}\text { Diagnostic performance of routine ultrasound screening for } \\
\text { fetal abnormalities in an unselected Swedish population in } \\
2000-2005\end{array}$ & Retrospective & Unselected & Two & $2000-2005$ & Repeat anomaly & $30-34$ \\
\hline $\begin{array}{l}\text { Abu-Rustum et al. } \\
(2010)^{31}\end{array}$ & $\begin{array}{l}\text { Role of first-trimester sonography in the diagnosis of } \\
\text { aneuploidy and structural fetal anomalies }\end{array}$ & Retrospective & Unclear & One & $2002-2009$ & Repeat anomaly & $32-35$ \\
\hline $\begin{array}{l}\text { Manegold et al. } \\
(2011)^{32}\end{array}$ & $\begin{array}{l}\text { Is a routine ultrasound in the third trimester justified? } \\
\text { Additional fetal anomalies diagnosed after two previous } \\
\text { unremarkable ultrasound examinations }\end{array}$ & Prospective & Unclear & One & $1998-2008$ & Not stated & $28-32$ \\
\hline $\begin{array}{l}\text { Grande et al. } \\
(2012)^{33}\end{array}$ & $\begin{array}{l}\text { First-trimester detection of structural abnormalities and the } \\
\text { role of aneuploidy markers }\end{array}$ & Prospective & Low risk & One & $2002-2009$ & Not stated & $32-35$ \\
\hline Wang et al. & $\begin{array}{l}\text { Ultrasound screening of fetal structural abnormalities by } \\
\text { standard ultrasound views during the first trimester }\end{array}$ & Prospective & Unclear & One & $2008-2011$ & Not stated & $28-32$ \\
\hline
\end{tabular}

This article is protected by copyright. All rights reserved 
Skråstad et al.

$(2013)^{35}$

Vijaykumar et al.

$(2017)^{36}$

Ficara et al.

$(2020)^{22}$

Drukker et al

$(2020)^{21}$
A randomized controlled trial of third-trimester routine ultrasound in a non-selected population

Detection of structural fetal anomalies in third trimester which usually remains undetected in second trimester

Value of routine ultrasound examination at 35-37 weeks' gestation in diagnosis of fetal abnormalities

How often do we incidentally find a fetal abnormality at the

routine third-trimester growth scan? A population-based study
Randomized trial,

study group only

Prospective

Prospective

Retrospective
Unselected

Unclear

Unselected

Unselected
One

One

Two

Two
1989-1992

Repeat anomaly

(study group only)

unclear

Repeat anomaly

2014-2019

Repeat anomaly

2016-2018

This article is protected by copyright. All rights reserved 
Table 2 Number of fetuses affected by malformation(s) according to detection time and prevalence of third-trimester malformations

\begin{tabular}{|c|c|c|c|c|c|}
\hline \multirow[t]{2}{*}{ Study } & \multirow{2}{*}{$\begin{array}{l}\text { Fetuses scanned at } \\
\text { the third-trimester } \\
\text { (number) }\end{array}$} & \multirow{2}{*}{$\begin{array}{l}\text { Abnormalities detected } \\
\text { before the third- } \\
\text { trimester (number) }\end{array}$} & \multicolumn{2}{|c|}{ Abnormalities detected at the third-trimester } & \multirow{2}{*}{$\begin{array}{c}\text { Abnormalities } \\
\text { detected after birth } \\
\text { (number) }\end{array}$} \\
\hline & & & $\begin{array}{l}\text { Number } \\
\text { (percent) }\end{array}$ & $\begin{array}{l}\text { Prevalence per } 1000 \text { scans } \\
\text { (95\% Confidence interval) }\end{array}$ & \\
\hline Brocks et al. (1991) ${ }^{26}$ & 10752 & $\mathrm{~N} / \mathrm{A}$ & 24 & $2.23(1.42-3.22)$ & 0 \\
\hline Crane et al. (1994) ${ }^{27}$ & 7575 & 31 & $34(19.5 \%)$ & $4.49(3.10-6.13)$ & 109 \\
\hline Hernádi et al. (1997) ${ }^{28}$ & 3936 & 44 & $10(15.6 \%)$ & $2.54(1.17-4.39)$ & 10 \\
\hline Eik-Nes et al. (2000) ${ }^{29}$ & 808 & 2 & 0 & $0(0.00-2.13)$ & 17 \\
\hline Romosan et al. (2009) ${ }^{30}$ & 16775 & 160 & $80(13.9 \%)$ & $4.77(3.78-5.87)$ & 336 \\
\hline Abu-Rustum et al. $(2010)^{31}$ & 1370 & 34 & $2(5.6 \%)$ & $1.46(0.02-4.39)$ & 0 \\
\hline Manegold et al. (2011) ${ }^{32}$ & 5044 & 218 & $44(15.2 \%)$ & $8.72(6.33-11.49)$ & 27 \\
\hline Grande et al. $(2012)^{33}$ & 13723 & N/A & 103 & $7.51(6.13-9.02)$ & Not stated \\
\hline Wang et al. $(2013)^{34}$ & 2822 & 22 & 1 & $0.35(0-1.52)$ & Not stated \\
\hline Skråstad et al. (2013) ${ }^{35}$ & 3175 & 22 & $18(25.7 \%)$ & $5.67(3.32-8.61)$ & 30 \\
\hline Vijaykumar et al. $(2017)^{36}$ & 10000 & N/A & 37 & $3.70(2.60-4.99)$ & 0 \\
\hline Ficara et al. $(2020)^{22}$ & 52713 & 674 & $247(24.8 \%)$ & $4.69(4.12-5.29)$ & 74 \\
\hline Drukker et al. $(2020)^{21}$ & 13023 & 288 & $43(9.1 \%)$ & $3.30(2.38-4.37)$ & 143 \\
\hline
\end{tabular}

Data are number or number (percent). For randomized trials, only the arm with routine third-trimester scan was selected 


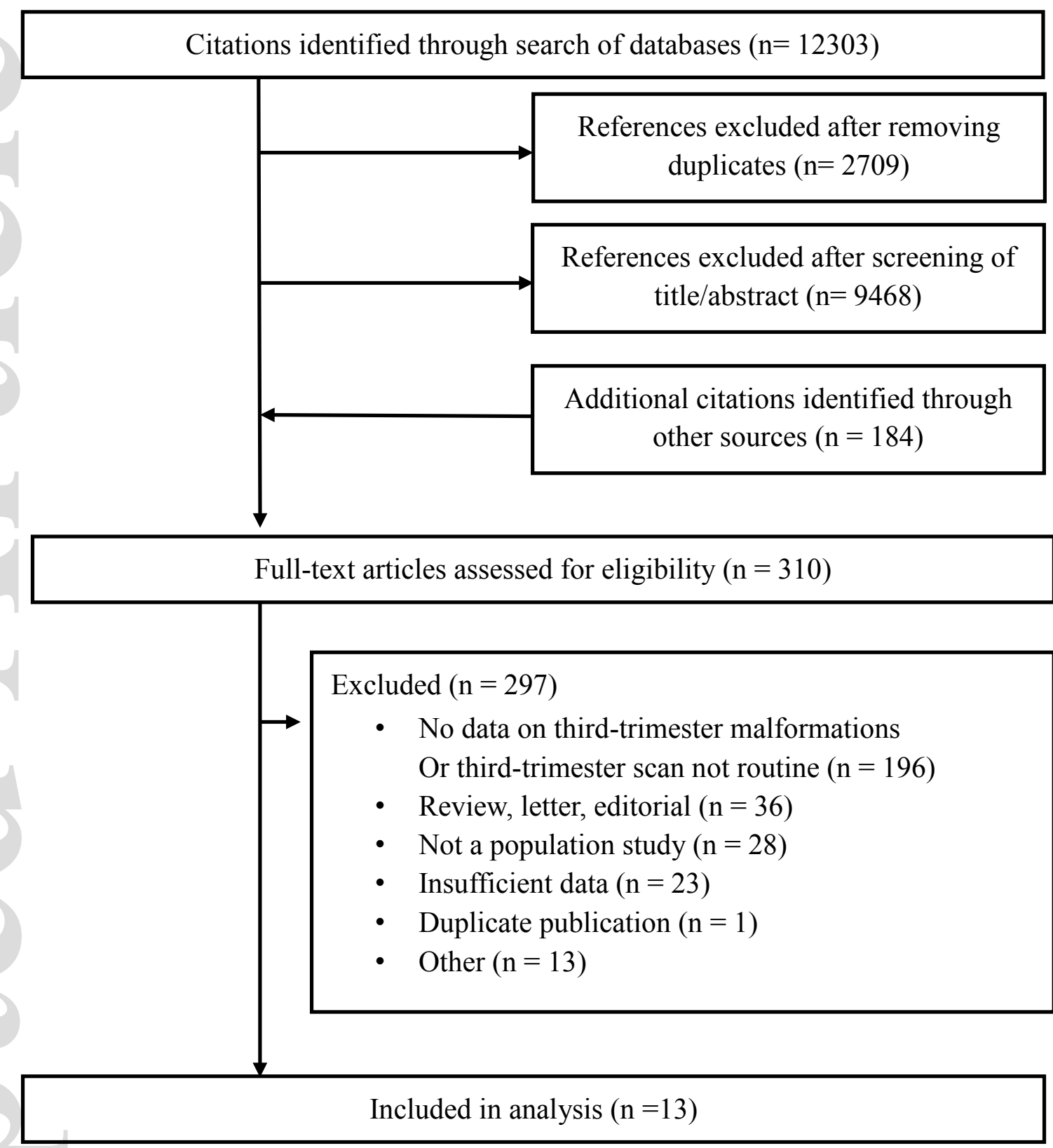

This article is protected by copyright. All rights reserved 
Study

Brocks et al. (1991)

Crane et al. (1994)

Hernádi et al. (1997)

Eik-Nes et al. (2000)

Romosan et al. (2009)

Abu-Rustum et al. (2010)

Manegold et al. (2011)

Grande et al. (2012)

Wang et al. (2013)

Skråstad et al. (2013)

Vijaykumar et al. (2017)

Ficara et al. (2020)

Drukker et al. (2020)

Random effects model

Heterogeneity: $I^{2}=88 \%, \tau^{2}=0.0002, \chi_{12}^{2}=97.14(p<0.01)$

10752

7575

3936

808

16775

1370
5044

13723

2822

3175

10000

52713

13023
Women attending Congenital Prevalence

scan (n) abnormality (n) (per 1,000) $95 \%$ Confidence Interval

(n) (per 1,

2.23

4.49
2.54

0.00

4.77

1.46

8.72

7.51
0.35

0.35

5.67

3.70
4.69

3.30

3.68

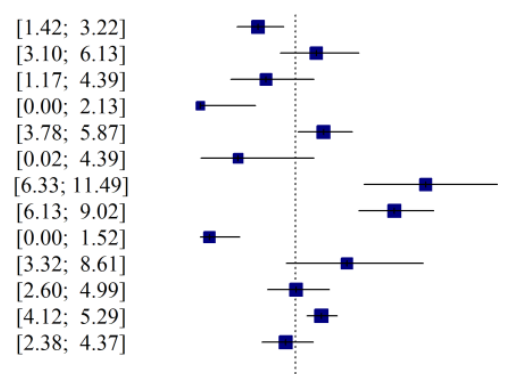

[2.72; 4.78]

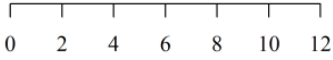

Prevalence of abnormalities detected in the third trimester

(per 1,000)

bjo_16468_f2.tif

This article is protected by copyright. All rights reserved 